\title{
Dentistry
}

\section{Maxillary Osteomyelitis by Mucormycosis: A Case Report and Literature Review}

Diljith Rishi, Akshay Shetty*, Nikhil Srivastava, Rajani BC, Pratyush Anshuman, Pragati Kakkar

Head of Department, Oral and Maxillofacial Surgery, Sri Rajiv Gandhi College of Dental Sciences and Hospital, Hebbal, Bangalore, Karnataka, India

\begin{abstract}
Mucormycosis is an acute infection which can manifest in many forms. The infection begins in the paranasal sinuses which goes on to affect the vasculature and leads to necrosis of hard and soft tissues. The most common presentation is the maxillary and orbital region in the area of head and neck. Here, we report a case of chronic osteomyelitis of the maxillary bone secondary to mycotic infection.
\end{abstract}

Keywords: Mucormycosis; Osteomyelitis; Necrosis; Aspergillosis

\section{Introduction}

Mucormycosis presents as an acute infection and manifests in rhino cerebral, pulmonary, gastrointestinal, cutaneous, or disseminated forms $[1,2]$. The infection begins in the upper turbinate or paranasal sinuses or less commonly in the palate or pharynx $[3,4]$. The most common presentation in maxillary and orbital cellulitis is in a person with inadequately controlled diabetes mellitus [5]. We describe here a case of chronic osteomyelitis of maxilla secondary to mycotic infections like mucormycosis and aspergillosis.

\section{Case Report}

A 60 year old male patient reported to the maxillofacial surgery department with a chief complaint of discharge from left nostril since 2 years. Patient gave a history of extraction of upper front teeth 2 years back due to swelling in that region; following the extraction the wound failed to heal and the patient started experiencing oral feeds coming out through the nose. Patient was subjected to a biopsy from the non healing wound 1 year back which was reported as mucormycosis. Patient did not take any treatment subsequently.

The patient's medical history showed diabetes mellitus and he was on medication. He also gave a history of tuberculosis and had undergone treatment 7 years back. On general examination vital signs were within normal limits. On extra oral examination there was no obvious facial asymmetry detected. On intra oral examination there was a breach in the labial mucosa and an exposed necrotic bone was present on the left maxilla extending from the left central incisor region to the left first molar region (Figure 1). On probing mobility was elicited in the necrotic bone. A provisional diagnosis of chronic osteomyelitis of secondary to mucormycosis was given.

A radiograph (orthopantomogram) was taken which showed bony defect in the maxillary anterior region till the premolar region and also showed haziness in the left maxillary sinus (Figure 2). A computed tomography (CT) scan of brain without contrast showed the defects in the lateral part of the body of the maxilla, anterior wall of maxilla and floor of maxillary sinus with oroantral and oro nasal communication (Figure 3 ). A chest $\mathrm{X}$ ray was taken which revealed multiple calcific foci in both upper and mid zones with few parenchymal bands. Biochemical investigations revealed a normal blood sugar levels. Fasting blood sugar was $90 \mathrm{mg} / \mathrm{dl}$ (normal 60-90 mg/dl) \& post prandial blood sugar level was $101 \mathrm{mg} / \mathrm{dl}$ (normal $90-140 \mathrm{mg} / \mathrm{dl}$ ).

An excisional biopsy was done. Cytological smear and PAS stain showed fungal infection predominantly mucormycosis organisms superimposed with few candida. Under high power PAS stain numerous septate (aspergillosis) and nonseptate (mucormycosis) hyphae were seen (Figures 4 and 5).

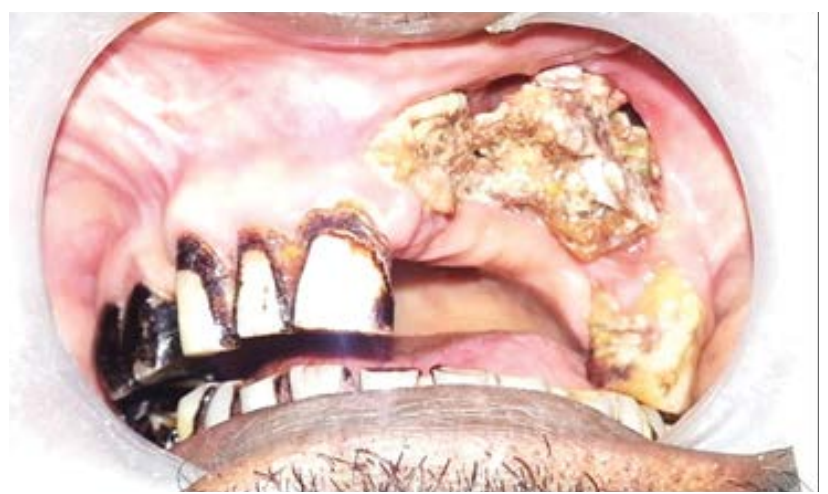

Figure 1: Depicting the exposed discoloured necrotic bone present on the left maxilla.

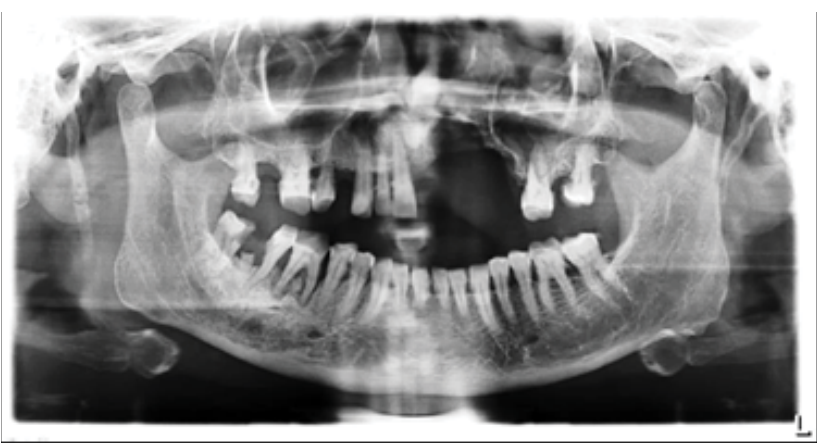

Figure 2: OPG showing bony defect in the maxillary anterior region till the premolar region and also showed haziness in the left maxillary sinus.

*Corresponding author: Akshay Shetty, Head of Department, Oral and Maxillofacial Surgery, Sri Rajiv Gandhi College of Dental Sciences and Hospital, Hebbal, Bangalore, Karnataka, India, Tel: +91-9845477547; E-mail: akshayshetty7978@gmail.com

Received July 12, 2018; Accepted July 16, 2018; Published July 22, 2018

Citation: Rishi D, Shetty A, Srivastava N, Rajani BC, Anshuman P, et al. (2018) Maxillary Osteomyelitis by Mucormycosis: A Case Report and Literature Review. Dentistry 8: 501. doi:10.4172/2161-1122.1000501

Copyright: (c) 2018 Rishi D, et al. This is an open-access article distributed under the terms of the Creative Commons Attribution License, which permits unrestricted use, distribution, and reproduction in any medium, provided the original author and source are credited. 
Citation: Rishi D, Shetty A, Srivastava N, Rajani BC, Anshuman P, et al. (2018) Maxillary Osteomyelitis by Mucormycosis: A Case Report and Literature Review. Dentistry 8: 501. doi:10.4172/2161-1122.1000501

The patient was hospitalized and under local anesthesia necrotic bone was mobilized with periosteal elevator and was removed (Figure 6). The patient was prescribed antibiotics amoxicillin and clavulanic acid and metronidazole for 5 days, analgesic diclofenac sodium for 3 days and antifungal itraconazole $200 \mathrm{mg}$ for 30 days. The excisional biopsy report showed deep mycotic infection predominantly aspergillosis with few mucormycosis organisms. Two weeks later the area started healing and subsequently after three months an obturator was made for the patient (Figure 7).

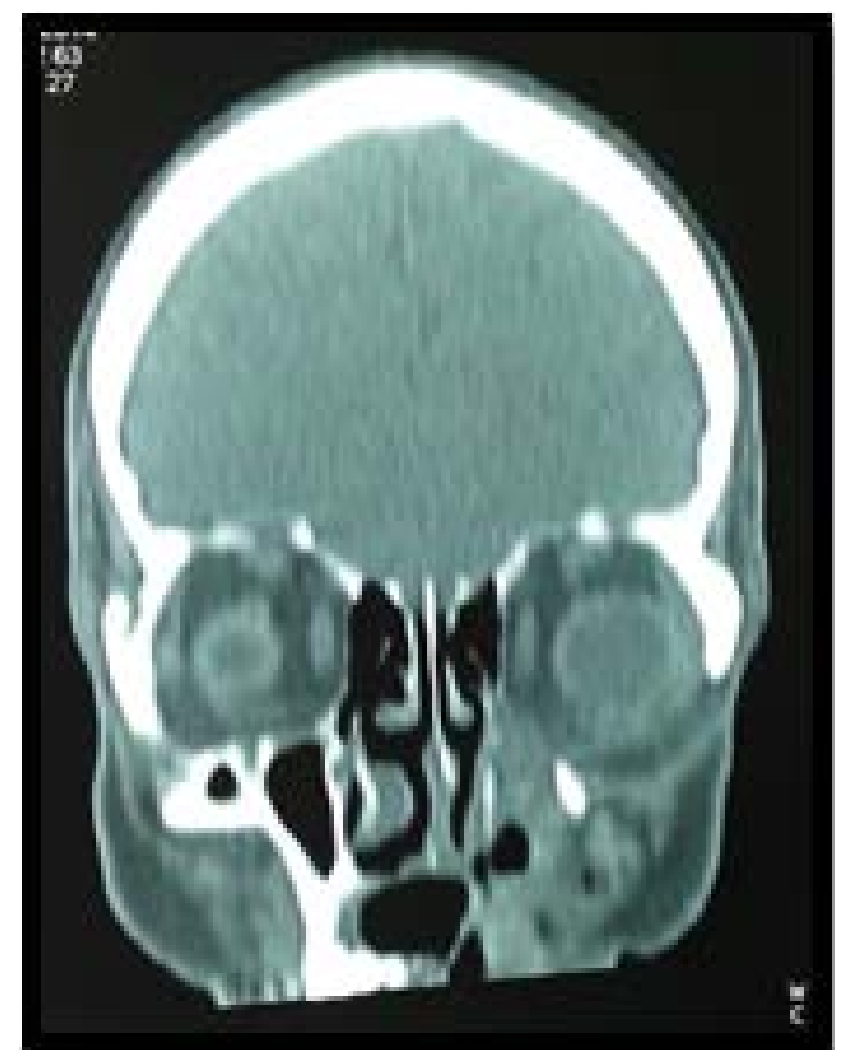

Figure 3: CT depicting the defects in the lateral part of the body of the maxilla anterior wall of maxilla and floor of maxillary sinus with oro antral and oro nasal communication.

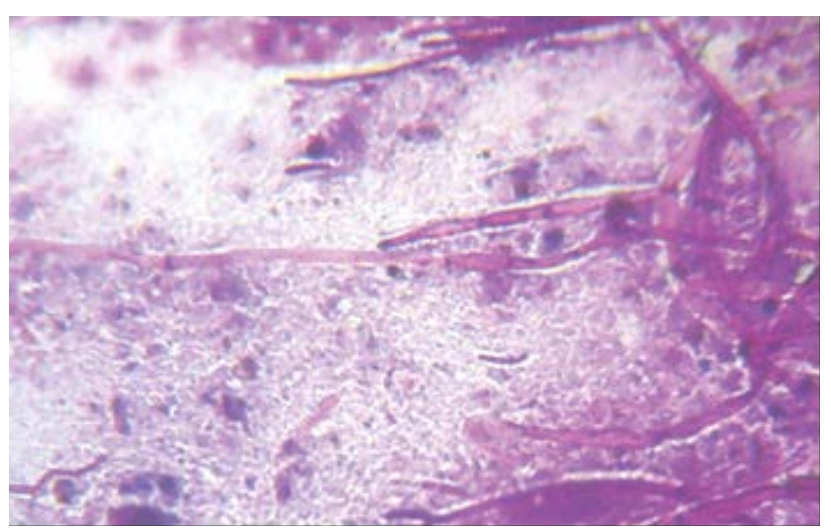

Figure 4: Under high power PAS stain numerous septate (aspergillosis) were seen.

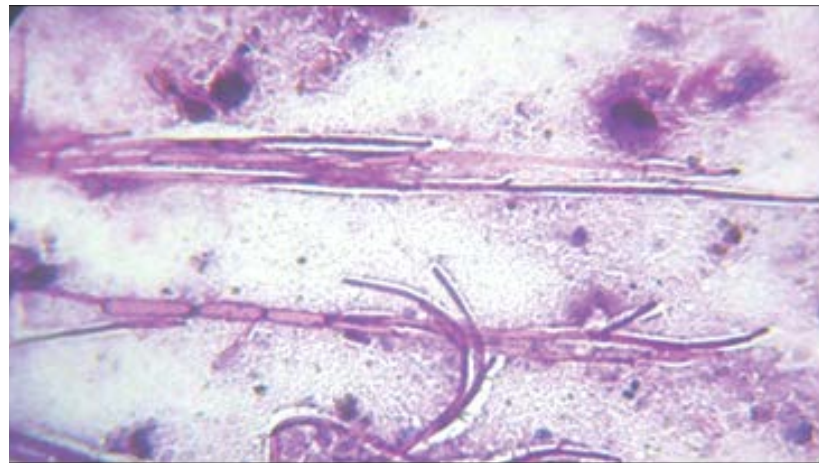

Figure 5: Under high power PAS stain nonseptate (mucormycosis) hyphae are seen.

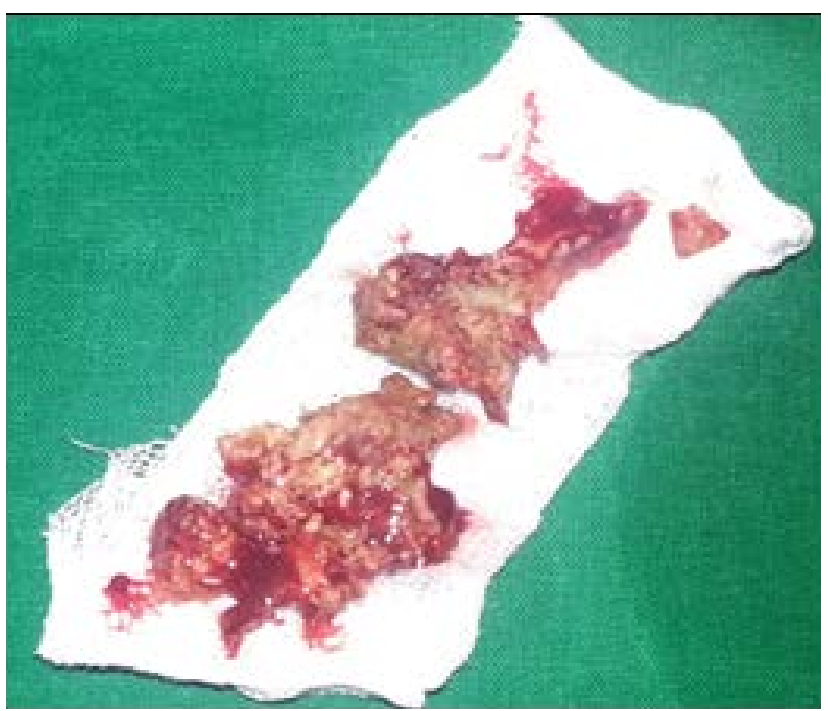

Figure 6: Showing the removed necrotized bone.

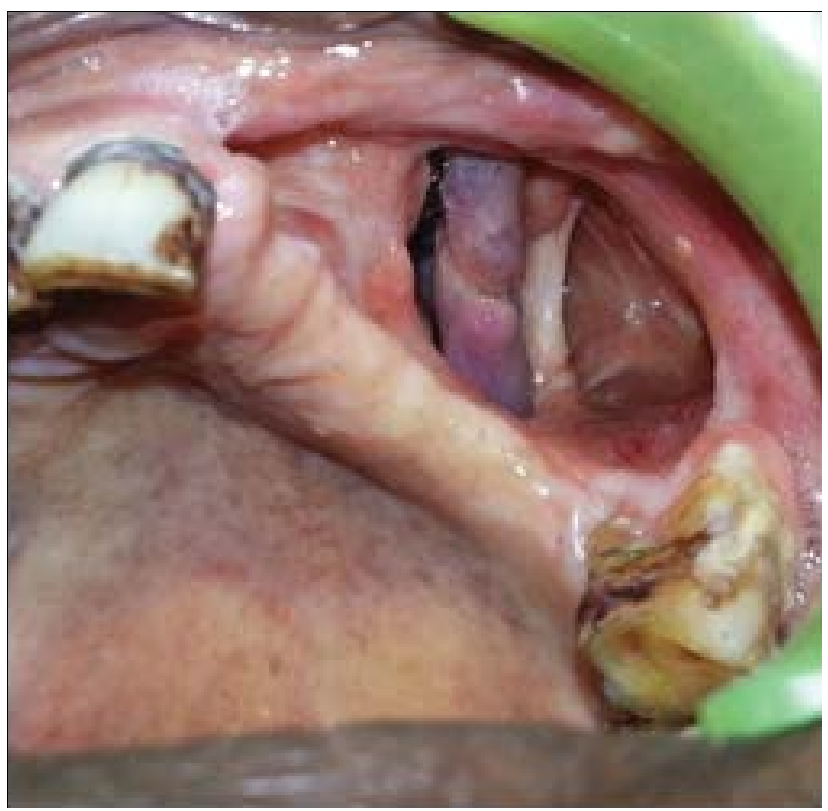

Figure 7: Showing one month post operation image. 
Citation: Rishi D, Shetty A, Srivastava N, Rajani BC, Anshuman P, et al. (2018) Maxillary Osteomyelitis by Mucormycosis: A Case Report and Literature Review. Dentistry 8: 501. doi:10.4172/2161-1122.1000501

\section{Discussion}

Mucormycosis is a rare filamentous fungal infection mostly encountered among immuno-suppressed patients [6]. Our patient had uncontrolled diabetes which is a well known predisposing factor for mucormycosis [7].

Presence of diabetic ketoacidosis is a condition which increases predisposition to mucormycosis. Acidosis disrupts iron binding of transferrin, resulting in increased proportion of unbound iron, which may promote growth of the fungus [8]. Uncontrolled diabetes mellitus can alter the normal immunologic response of patients to infections. Such patients have decreased granulocyte phagocytic ability with altered polymorphonuclear leukocyte response [9].

Although several genera are associated with this disease, the most common forms are Rhizopus, Rhizomucor and Absida. Rhizopus is the predominant pathogen accounting for $90 \%$ of the cases of rhinocerebral mucormycosis. This microbe may be cultured from the oral cavity, nasal passages, throat and stool of healthy patients without clinical signs of infection [9].

Typically mucormycosis occurs as a pulmonary, gastrointestinal, disseminated or rhinocerebral infection [10]. In our patient, infection was only localized to the maxilla and it underwent necrosis without any other symptoms. Disseminated involvement of mucormycosis is observed in diabetics with ketoacidosis, which favors rapid proliferation of fungus and its invasion into the orbit and cerebrum [11].

\section{Conclusion}

This report will help the practitioners in properly diagnosing and formulating a management plan for mucormycosis, and this rare case shows the variation in location, incidence of the lesion and also states various concomitant signs and symptoms. Mucormycosis is a rare infection and patients not exhibiting other disseminating infection can be managed effectively.

The authors declare that there is no conflict of interest regarding the publication of the paper.

\section{References}

1. Sugar AM (2005) Agents of mucormycosis is and related species. In: Mandell, Bennett, Dolin, editors. sixth ed., Mandell Douglas and Bennett's principles and practice of infectious diseases, sixth ed. Philadelphia, Pennsylvania USA Elsevier Churchill Livingstone 2: 2973-2984.

2. Leitner C, Hoffmann J, Zerfowski M, Reinert S (2003) Mucormycosis: necrotizing soft tissue lesion of the face. J Oral MaxillofacSurg 61: 1354-1358.

3. Abidi E, Sismanis KC, Pastore P (1984) Twenty-five years experience treating cerebro rhino orbital mucormycosis. Laryngoscope 94: 1060-1062.

4. Morduchowicz G, Schmueli D (1986) Rhinocerebral mucormycosis in renal transplant patients: report of 3 cases and review of literature. Rev Infect Dis 8: $444-446$.

5. Marx RE, Stern D (2006) Oral and maxillofacial pathology: a rationale for diagnosis and treatment. first ed., UK: Quintessence Publishing Co., Inc 104106.

6. Eucker J, Sezer O, Graf B, Possinger K (2001) Mucormycosis. Mycoses 44 253-260.

7. Auluck A (2007) Maxillary necrosis by mucormycosis. A case report and literature review. Med Oral Patol Oral Cir Bucal 12: e360-e364.

8. Sheldon $\mathrm{WH}$, Bauer $\mathrm{H}$ (1959) The development of the acute inflammatory response to experimental cutaneous mucormycosis in normal and diabetic rabbits. J Exp Med 110: 845-852.

9. Brown OE, Finn R (1986) Mucormycosis of the mandible. J Oral MaxillofacSurg 44: 132-136.

10. Napoli JA, Donegan JO (1991) Aspergillosis and necrosis of maxilla: a case report. J Oral MaxillofacSurg 49: 532-534.

11. Buhl MR, Joseph TP, Snelling BE, Buhl L (1992) Temporofacialzygomycosis in a pregnant woman. Infection 20: 230-232. 